\title{
Enhancement of Dunaliella salina growth by using wavelength shifting dyes
}

\author{
Hatice Burak ${ }^{1,2}$ - Alan Dunbar ${ }^{1} \cdot$ D. James Gilmour ${ }^{2}$ (D) \\ Received: 14 January 2019 / Revised and accepted: 3 May 2019 / Published online: 24 May 2019 \\ (C) The Author(s) 2019
}

\begin{abstract}
The availability of light is one of the key driving factors for photosynthesis. Chlorophyll and accessory pigments absorb light at some of the wavelengths present in the solar spectrum. If all other requirements are available in abundance, introducing more light to the system at wavelengths that can be absorbed will increase the growth rate of microalgae and the final biomass achieved up to the level of photosaturation. Therefore, the effect of shifting the spectrum of light available such that the photons unutilized in photosynthesis are converted to those that can be utilised was investigated. Spectral shifts converting UV light to blue light and green light to red light were carried out using films produced with Coumarin and Solvent Orange respectively. Dunaliella salina (CCAP 19-30) was grown in an airlift photobioreactor (ALB) which was coated with the wavelength shifting films. Dunaliella final biomass in the ALB, as determined by the optical density, were shown to increase by $36.9 \%$ for UV to blue light conversion and by $18.8 \%$ for green to red light conversion when using a coated ALB compared with an uncoated one.
\end{abstract}

Keywords Fluorescent dye $\cdot$ Wavelength shifting $\cdot$ Dunaliella salina $\cdot$ Airlift photobioreactor

\section{Introduction}

Photoautotrophic growth of microalgae utilising $\mathrm{CO}_{2}$ as the sole source of carbon and sunlight for energy is an attractive system for the production of agriculture and aquaculture foods and high-value bioactives, complex oils, hydrocarbons and lipids, and potentially biofuels (Melis 2002; Gushina and Harwood 2006; Chisti 2010). Algal growth depends on photosynthesis which requires light, inorganic salts, water and carbon dioxide, in conjunction with appropriate levels of salinity, $\mathrm{pH}$ and temperature.

$6 \mathrm{CO}_{2}+6 \mathrm{H}_{2} \mathrm{O} \underset{\text { Light }}{\rightarrow} \mathrm{C}_{6} \mathrm{H}_{12} \mathrm{O}_{6}+6 \mathrm{O}_{2} \quad\left(T=20-30^{\circ} \mathrm{C}\right)$

Electronic supplementary material The online version of this article (https://doi.org/10.1007/s10811-019-01819-4) contains supplementary material, which is available to authorized users.

D. James Gilmour

D.J.Gilmour@Sheffield.ac.uk

1 Department of Chemical and Biological Engineering, The University of Sheffield, Mappin Street, Sheffield S1 3JD, UK

2 Department of Molecular Biology and Biotechnology, The University of Sheffield, Western Bank, Sheffield S10 2TN, UK
Oxygen is generated during photosynthesis and an excess of free $\mathrm{O}_{2}$ inhibits algal growth; therefore, $\mathrm{O}_{2}$ accumulation must be controlled (Molina et al. 2001). Aeration systems which produce fine bubbles are beneficial when used inside photobioreactors (Zimmerman et al. 2011 ) in order to expedite the removal of excess $\mathrm{O}_{2}$ accumulation.

Photosynthetically active radiation (PAR) is defined as light between 400 and $700 \mathrm{~nm}$ (Kirk 1994; Chen and Blankenship 2011). The light required for photosynthesis is absorbed by light harvesting chlorophyll pigments. These pigments have absorption peaks at $450 \mathrm{~nm}$ (blue light region) and $680 \mathrm{~nm}$ (red light region) and accessory pigments such as carotenoids typically have absorption peaks around 450-530 $\mathrm{nm}$. The light absorbed by light harvesting chlorophyll molecules and accessory pigments is passed on to special chlorophyll reaction centre molecules (photosystems I and II) and used to drive photosynthetic electron transport and hence the production of ATP and NADPH (Carvalho et al. 2011). There is little or no absorption between 530 and $620 \mathrm{~nm}$ which corresponds to green, yellow and orange light. Hence, artificial light can be used to grow microalgae under illumination at the exact wavelength range absorbed; however, it will be very costly (Molina Grima et al. 1999; Acien Fernandez et al. 2013). Sunlight is the cheapest available light source for 
algal growth and is comprised of a broad range of emission wavelengths. Shifting the sunlight to the specific wavelengths used in photosynthesis will increase the growth efficiency (up to the photosaturation limit) assuming all other requirements, e.g. $\mathrm{CO}_{2}$, nutrients, water, are supplied in abundance and also that $\mathrm{O}_{2}$ removal is optimised. It should be noted that sunlight contains UV radiation (harmful to algae) as well as PAR; therefore, the work described in this paper used organic dyes to convert photosynthetically unused UV and green light to photosynthetically useful blue and red light, respectively.

A good wavelength shifting material will efficiently absorb the unused wavelengths of sunlight and re-emit this absorbed energy as light at wavelengths which overlap with chlorophyll and carotenoid absorption bands. When choosing wavelength shifting materials, some criteria must be taken into consideration such as (1) absorption wavelengths that only absorb unused light; (2) emission wavelength range which will overlap with the algal cell's absorbance wavelengths; (3) quantum efficiency of the emission from the material; (4) reflection and refraction indexes of the material; (5) cost of production; (6) decomposition of the material over time (Hovel et al. 1979; Van Sark et al. 2005; Klampaftis et al. 2009).

Wondraczek et al. (2013) studied the conversion of green light into red light using $\mathrm{Ca}_{0.59} \mathrm{Sr}_{0.40} \mathrm{Eu}_{0.01} \mathrm{~S}$ as a wavelength shifting material and they achieved $>20 \%$ spectral conversion of unused green light into red light. Furthermore, Uyar et al. (2007) used Rhodamine B solution and $\mathrm{CuSO}_{4}$ solution to shift wavelengths $>760 \mathrm{~nm}$ and $<630 \mathrm{~nm}$, respectively. In their study of hydrogen production, they succeed in enhancing the light intensity by $180 \mathrm{~W} \mathrm{~m}^{-2}$. Also, Delavari Amrei et al. (2014) studied the biomass productivity of Chlorella sp. (PTCC 6010) using Uvitex OB, and they succeeded to increase the productivity by $10 \%$. In keeping with this previous research on wavelength shifting materials, the work presented here considers the influence of two different wavelength shifting materials on the growth of Dunaliella salina CCAP 19/30 in an ALB. Dunaliella was chosen as the algae species because of its high $\mathrm{CO}_{2}$ uptake properties (Ying et al. 2013). In addition, $D$. salina CCAP19/30 strain was chosen because it does not produce high amounts of $\beta$-carotene in contrast to other strains of $D$. salina such as CCAP19/18 (Olmos et al. 2009). Growth was carried out under $24 \mathrm{~h}$ exposure to a combination of UV light and white light transmitted in order to mimic sunlight. The illumination passed through two different wavelength shifting materials so as to convert the UV wavelengths to blue light and green to red.

The purpose of this research was to develop an ALB design incorporating wavelength shifting technology in order to increase algal biomass production through the application of wavelength shifting materials that increase the amount of usable light.

\section{Materials and methods}

\section{Fabrication of wavelength shifting materials}

Wavelength shifting films were produced using two different organic dyes Coumarin 1 purchased from Sigma Aldrich Ltd. and Bestoil Orange 2G (commonly called Solvent Orange 63) purchased from Fast Colour Ltd. Both dyes were dissolved in tetrahydrofuran (THF) and encapsulated in polydimethylsiloxane (PDMS) (SYLGARD) 184 silicone elastomer matrix. The PDMS was supplied by Dow Corning as two constituents which are the base polymer and a curing agent which were mixed in a fix base/curing agent ratio (10/1) by weight. Firstly, a solution of the required organic dye (Coumarin or Solvent Orange) was prepared at a concentration of $1 \mathrm{mg} \mathrm{mL}^{-1}$. Once the fluorescent dye was completely dissolved in the THF, it was mixed with the PDMS. Typically, $25 \mathrm{~g}$ of the PDMS base was put into a beaker with $2.5 \mathrm{~g}$ of the curing agent and then $25 \mathrm{~g}$ of the fluorophore solution was added to the liquid PDMS. The mixtures were then mixed rigorously to obtain a very smooth sticky paste and any large bubbles were burst using a small spatula. The paste was poured onto a glass mould $(268 \times 175 \mathrm{~mm}$ and $1 \mathrm{~mm}$ deep) and any remaining bubbles were burst. Next, the mould was laid horizontally in oven at $80^{\circ} \mathrm{C}$ for $24 \mathrm{~h}$. After $24 \mathrm{~h}$, a scalpel was used to detach the cured rubbery PDMS film, which was subsequently peeled out of the mould.

\section{Optical characterisation of the films}

The UV-visible absorbance spectra were measured using an Ocean Optics USB2000 spectrometer and a Mikropack MiniD2 UV-vis-IR light source, and data was recorded and analysed using SpectraSuite software. The emission spectra of the wavelength shifting films were measured using a FluoroMax 4 spectrofluorometer from HORIBA Scientific.

\section{Choice of dyes}

The choice of dyes was made based on their absorbance and emission characteristics. Coumarin absorbs UV light and emits light in the blue region of the spectrum (see Fig. 2). Solvent Orange absorbs in the green part of the spectrum and emits in the orange/red part of the spectrum. Both dyes absorb high energy photons and emit at lower energies.

\section{Growth of Dunaliella salina}

Dunaliella salina CCAP 19/30 was supplied by the Culture Collection of Algae and Protozoa (CCAP), Oban, UK. The growth medium for Dunaliella, developed by Hard and Gilmour (1996), was used. Approximately $200 \mathrm{~mL}$ of freshly subcultured $D$. salina cells was added to the reactor until an 
initial optical density of 0.068 at $595 \mathrm{~nm}$ was achieved. The same initial optical density was used for all experiments. After recording the initial optical density at $595 \mathrm{~nm}$, the reactors were placed inside the purpose built growth chamber (Fig. 1). The growth chamber served two purposes: firstly, it ensured no external light could enter the chamber; secondly, it prevented temperature variation inside the chamber. The growth chamber contained a UV lightbox and a white lightbox. The UV lightbox contained two Sylvania Blacklight 368 (F20W/T12/BL368) UVA lamps and the white lightbox contained two Sylvania Fluorescent Cool White lamps (F20W/33-640/IRS). The spectral output from these lamps is given in supplementary information Figure S1. A Forston Labs LabNavigator temperature probe was used to monitor the air temperature inside the chamber.

Four separate experiments, control 1, control 2, UV to blue wavelength shifting and green to red wavelength shifting, were run. Control 1 was a photobioreactor (PBR) without any wavelength shifting film exposed to both UV and white light in order to determine the influence of the UV and white light illumination on the microalgae cells, whereas control 2 was just illuminated with white light. Both UV and white light were used for the UV to blue wavelength shifting experiment with the coating applied to UV light side only. Finally, the green to red wavelength shifting experiment was conducted with the coated side facing the white light, with a green to red wavelength shifting film exposed to white light only (Figs. 1 and 2).

As shown in Fig. 1, the reactors were connected to an air pump to supply fine bubbles in order to deliver $\mathrm{CO}_{2}$ and prevent $\mathrm{O}_{2}$ accumulation. In order to measure the growth, 1-mL samples were taken every $24 \mathrm{~h}$ to obtain the growth pattern of the microalgae. The emission spectra of the illumination used are given in Fig. 3 and it is clear that the UV light emits $\mathrm{UV}<400 \mathrm{~nm}$ and also four narrow peaks of visible light

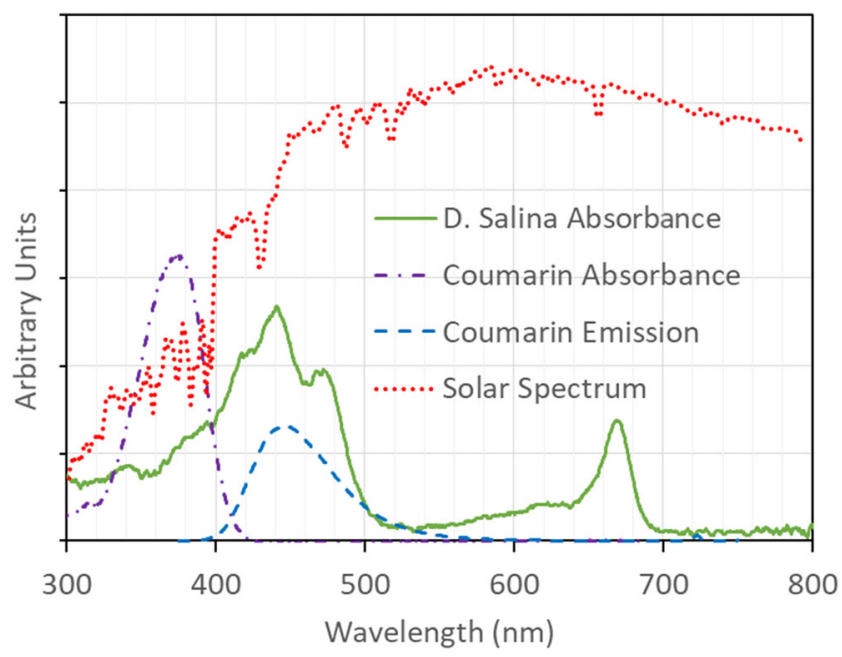

Fig. 2 A graphical representation of the wavelength shifting concept showing the absorbance (AU) and emission spectra (photons per second) of the Coumarin dye showing the shift in wavelengths between UV and blue light. The solar emission spectrum (in photons $\mathrm{m}^{-2} \mathrm{~s}^{-1}$ ) and the Dunaliella salina absorbance spectrum (AU) are also shown. Each dataset has been arbitrarily scaled so they can all be presented on the same figure

at wavelengths $>400 \mathrm{~nm}$ which will influence the growth pattern of control 1 experiment.

\section{Results and discussion}

UV to blue wavelength shifting thin films and green to red wavelength shifting thin films were used for this experiment. Figure 3 indicates the transmission properties of the wavelength shifting film-coated reactors compared with the absorbance of D. salina CCAP 19-30. It is clear from Fig. 3 that for the UV to blue wavelength shifting film, it absorbs at
Fig. 1 Experimental set-up for Dunaliella salina growth (a). The growth chamber design showing the dark enclosure with access and baffles to allow air circulation. Note the black walls have been shown as transparent and there is one light box in front of the ALB and another lightbox behind (b). A plan view of the reactor and light sources within the growth chamber. For control experiments, no wavelength shifting materials were present

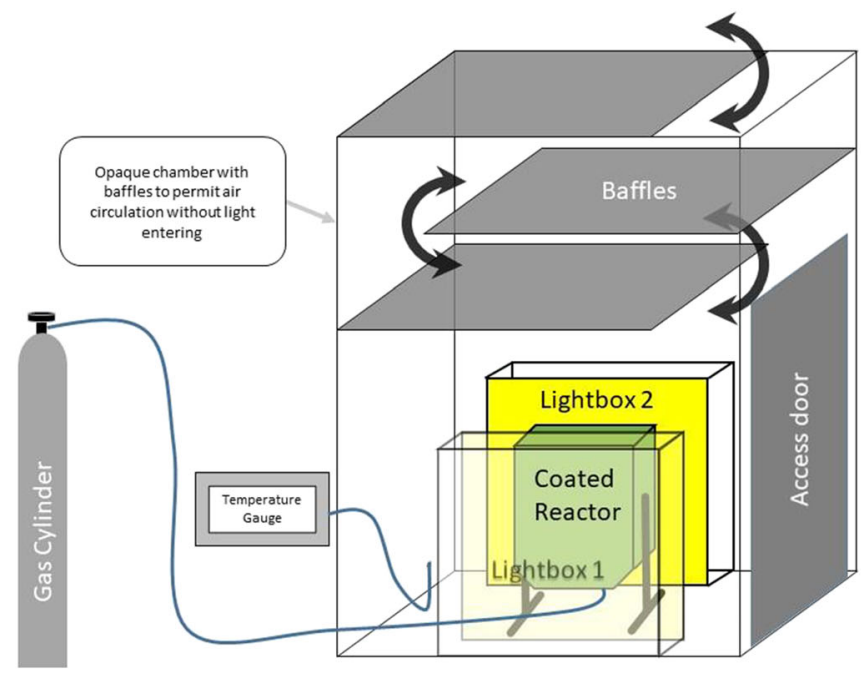

a

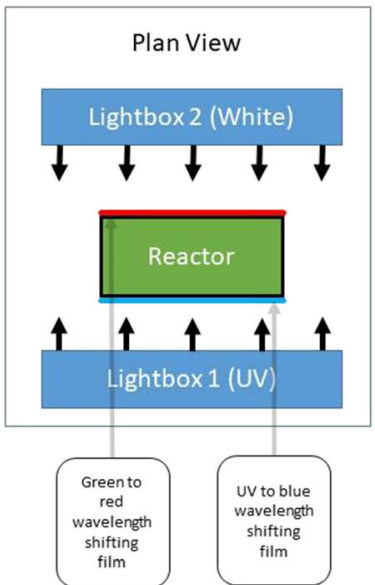

b 
Fig. 3 Wavelength shifting films used. UV to blue wavelength shifting fluorescent dye (a) and green to red wavelength shifting fluorescent dye (b). Optical micrograph taken with a $\times 40$ objective lens of the green to red wavelength shifting material (Labophot, Nikon) (c). Light transfer from the $3 \mathrm{~L}$

photobioreactor with and without wavelength shifting materials (d)
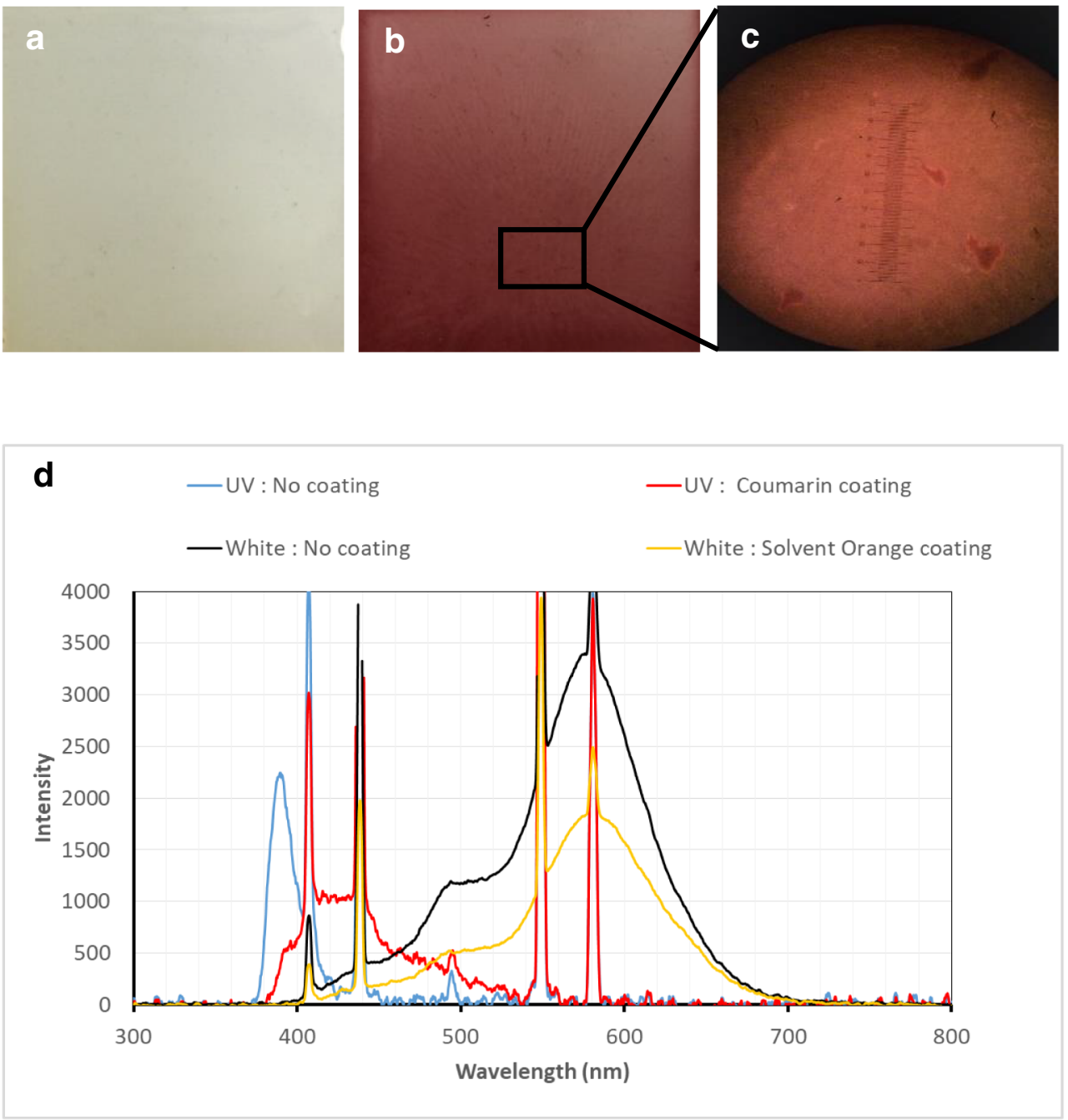

wavelengths $<420 \mathrm{~nm}$ which are unused by the algae and that light is subsequently re-emitted in a band that overlaps well with the absorption spectrum of the $D$. salina. This means that more photons will be absorbed by the microalgae cells, which will result in an enhancement of their growth. On the other hand, the green to red wavelength shifting thin film has less intense absorption features and it is superimposed on a background of scattering observed at all wavelengths (data not shown). This scattering of incident light is likely responsible for the reduced algal growth observed when using this film compared with the UV to blue wavelength shifting film. Less light overall will be entering the ALB. Furthermore, the reduced absorbance compared with the UV film will mean fewer photons will be captured by this green to red film and shifted to longer wavelength. Therefore, the spectra in Fig. 3 indicate that the UV to blue film should be more effective at increasing algal growth.

Figure 4 shows the growth pattern achieved with each setup and provides proof that wavelength shifting actively assists algal growth. The maximum OD values recorded in the latter stages of the growth patterns were 1.048, 0.814, 0.173 and 0.661 at $595 \mathrm{~nm}$ for UV to blue wavelength shifting film, green to red wavelength shifting film and control 1 and control 2 , respectively. The doubling times were calculated as 5.59, 5.97, 7.29 and 6.66 days respectively. These results mean that in comparison with control 2 , there is $39.9 \%$ and $18.8 \%$ increase in the final OD growth increments for UV to blue wavelength shifting and green to red wavelength shifting, respectively. In comparison with two other studies on wavelength shifting materials in the literature, one succeeded in reaching $>20 \%$ spectral conversion for green to red wavelength shifting (Wondraczek et al. 2013), and the other reached a $10 \%$ enhancement in biomass using commercial UV to blue wavelength shifting material (Delavari Amrei et al. 2014). Compared with these studies, this work has achieved greater microalgae production for the UV to blue wavelength shifting as indicated by the high final OD presented as a result of the high cell density in the algal culture (see Fig. 5). It should also be noted in Fig. 4 that for control 1 when both UV and white light are used, the presence of the UV 


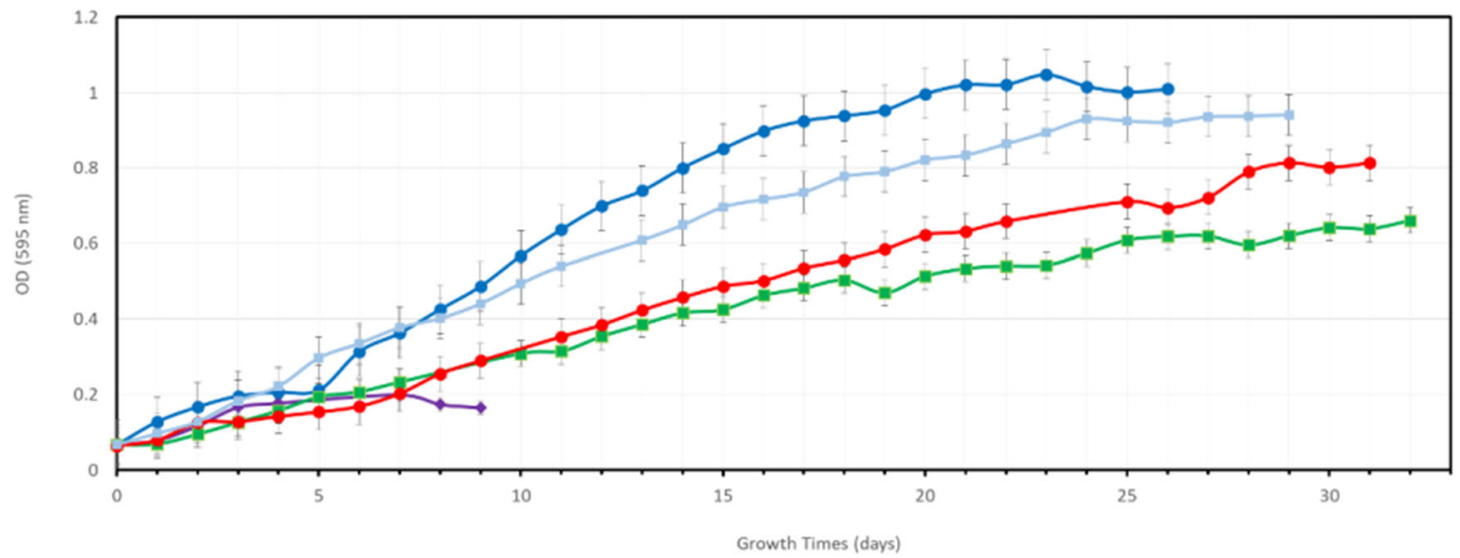

Fig. 4 Growth curves of the control experiments and when using coated reactors. Control 1 is without any wavelength shifting material but using both UV and white light in order to see the UV light effect which causes cell death in a few days; control 2 is without any wavelength shifting materials but using only white light; the UV to blue wavelength shifting experiments were done only with Coumarin 1 material and both the UV and white lights on; the green to red wavelength shifting experiments were done using only Solvent Orange material with only the white light on; and for the final combined experiments both Coumarin 1 and Solvent Orange materials were used with both lights on inhibits algae growth and causes cell death even when white light is also present. The UV light clearly has a detrimental effect on growth, but in the presence of the UV to blue shifting film there is clearly a protective effect against UV light. On the other hand, if only white light is used as in the control 2 experiment, a final biomass of about $0.661 \mathrm{OD}_{595}$ is obtained on the final day but the growth time is longer than for the other experiments with the wavelength shifting films. The photographs in Fig. 5 show the variation in optical density due to Dunaliella cells in each experimental run, the first and final day images of the reactors are shown. The initial images are all similar but the experiments with UV to blue wavelength shifting films (b) and (d) resulted in denser media on the final day compared with control 2 (a) and the green to red wavelength shifting experiments (c).
Fig. 5 Experiments on the first and final days of recorded algae growth. a Control 2. b UV to blue wavelength shifting. c Green to red wavelength shifting. $\mathbf{d}$ Both $\mathrm{UV}$ to blue and green to red wavelength shifting a
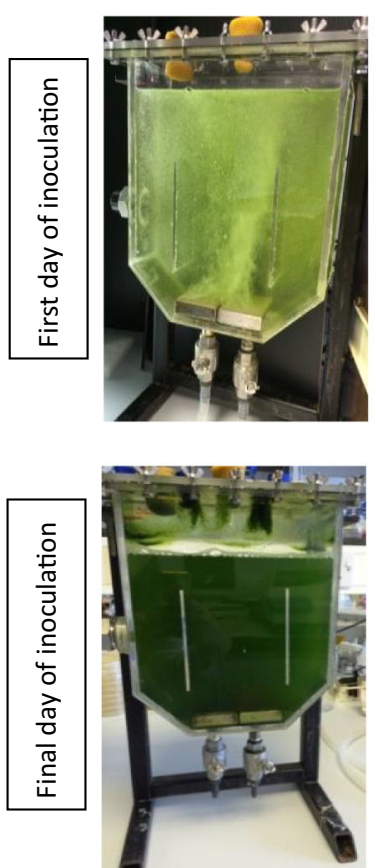

b
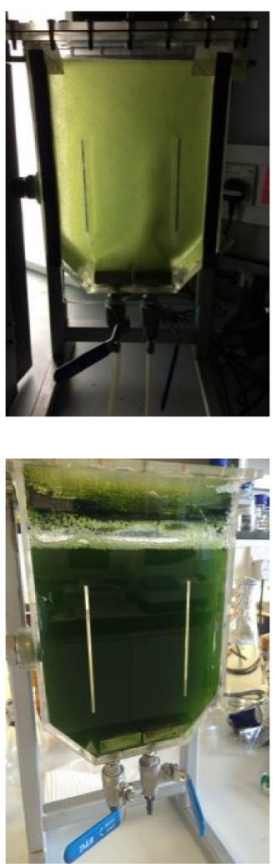

C
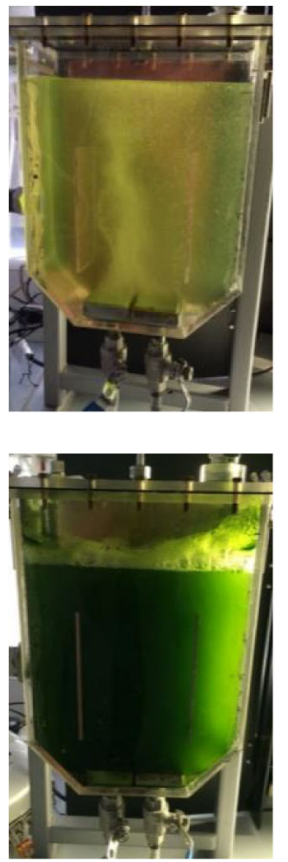

d
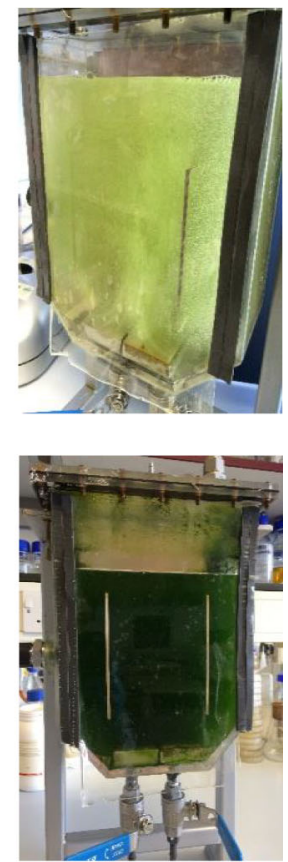


\section{Conclusion}

By introducing wavelength shifting technology to the ALB growth system, the optical densities of algal growth finally achieved were increased by $36.9 \%$ and $18.8 \%$ with UV to blue light conversion and green to red light conversion, respectively. The growth rate of the algae is also increased upon application of the Coumarin coating; with the Coumarin coating in place the growth profile plateaus at a maximum OD after about 20 days whereas the corresponding control (control 2) takes approximately 30 days to reach its maximum OD. Therefore, wavelength shifting technology offers a distinct enhancement allowing faster growth and denser algae cell cultures to be achieved.

Acknowledgements The authors wish to acknowledge Prof David Lidzey for access to the FluoroMax 4 spectrofluorometer.

Open Access This article is distributed under the terms of the Creative Commons Attribution 4.0 International License (http:// creativecommons.org/licenses/by/4.0/), which permits unrestricted use, distribution, and reproduction in any medium, provided you give appropriate credit to the original author(s) and the source, provide a link to the Creative Commons license, and indicate if changes were made.

\section{References}

Acien Fernandez FG, Fernandez Sevilla JM, Molina Grima E (2013) Photobioreactors for the production of microalgae. Rev Environ Sci Biotechnol 12:131-151

Carvalho AP, Silva SO, Baptista JM, Malcata FX (2011) Light requirements in microalgal photobioreactors: an overview of biophotonic aspects. Appl Microbiol Biotechnol 89:1275-1288

Chen M, Blankenship RE (2011) Expanding the solar spectrum used by photosynthesis. Trends Plant Sci 16:427-431

Chisti Y (2010) Fuels from microalgae. Biofuels 1:233-235

Delavari Amrei H, Ranjbar R, Rastegar S, Nasernejad B, Nejabebrahim A (2014) Using fluorescent material for enhancing microalgae growth rate in photobioreactors. J Appl Phycol 49:324-331

Gushina IA, Harwood JL (2006) Lipids and lipid metabolism in eukaryotic algae. Prog Lipid Res 45:160-186
Hard BC, Gilmour DJ (1996) The uptake of organic compounds by Dunaliella parva CCAP 19/9. Eur J Phycol 31:217-224

Hovel HJ, Hodgson RT, Woodall JM (1979) The effect of fluorescent wavelength shifting on solar cell spectral response. Sol Energy Mater 2:19-29

Kirk JT (1994) Light and photosynthesis in aquatic ecosystems. Cambridge University Press, Cambridge

Klampaftis E, Ross D, McIntosh KR, Richards BS (2009) Enhancing the performance of solar cells via luminescent down-shifting of the incident spectrum: a review. Sol Energy Mater Sol Cells 93:11821194

Melis A (2002) Green alga hydrogen production: progress, challenges and prospects. Int J Hydrog Energy 27:1217-1228

Molina Grima E, Fernandez J, Acien FG, Garcia Camacho F, Chisti Y (1999) Photobioreactors: light regime, mass transfer, and scaleup. J Biotechnol 70:231-247

Molina E, Fernandez J, Acien FG, Chisti Y (2001) Tubular photobioreactor design for algal cultures. J Biotechnol 92:113-131

Olmos J, Ochoa L, Paniagua-Michel J, Contreras R (2009) DNA fingerprinting differentiation between $\beta$-carotene hyperporoducer strains of Dunaliella from around the world. Saline Systems 5:5

Uyar B, Eroglu I, Yucel M, Gunduz U, Turker L (2007) Effect of light intensity, wavelength and illumination protocol on hydrogen production in photobioreactors. Int J Hydrog Energy 32:4670-4677

Van Sark WGJHM, Meijerink A, Schropp REI, Van Roosmalen JAM, LYSEN EH (2005) Enhancing solar cell efficiency by using spectral converters. Sol Energy Mater Sol Cells 87:395-409

Wondraczek L, Batentschuk M, Schmidt MA, Borchardt R, Scheiner S, Seemann B, Schweizer P, Brabec CJ (2013) Solar spectral conversion for improving the photosynthetic activity in algae reactors. Nat Commun 4:2047

Ying K, Gilmour DJ, Shi S, Zimmerman WB (2013) Growth enhancement of Dunaliella salina by microbubble induced airlift loop bioreactor (ALB) - the relation between mass transfer and growth rate. J Biomater Nanobiotechnol 4:1-9

Zimmerman WB, Zandi M, Bandulasena HCH, Tesar V, Gilmour DJ, Ying K (2011) Design of an airlift loop bioreactor and pilot scales studies with fluidic oscillator induced microbubbles for growth of a microalga Dunaliella salina. Appl Energy 88:3357-3369

Publisher's note Springer Nature remains neutral with regard to jurisdictional claims in published maps and institutional affiliations. 